\title{
openheart Amlodipine in hypertension: a first-line agent with efficacy for improving blood pressure and patient outcomes
}

\author{
Hassan Fares, ${ }^{1}$ James J DiNicolantonio, ${ }^{2}$ James H O’Keefe, ${ }^{2}$ Carl J Lavie ${ }^{1}$
}

To cite: Fares $\mathrm{H}$,

DiNicolantonio JJ,

O'Keefe JH, et al. Amlodipine in hypertension: a first-line agent with efficacy for improving blood pressure and patient outcomes. Open Heart 2016;3:e000473. doi:10.1136/openhrt-2016000473

Received 2 June 2016 Revised 10 August 2016 Accepted 15 August 2016

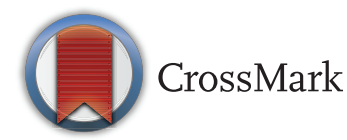

${ }^{1}$ Department of

Cardiovascular Diseases, John Ochsner Heart and Vascular Institute, Ochsner Clinical School-The University of Queensland School of Medicine, New Orleans, Louisiana, USA

${ }^{2}$ Saint Luke's Mid America Heart Institute, Kansas City, Missouri, USA

Correspondence to Dr James J DiNicolantonio; jjdinicol@gmail.com

\section{ABSTRACT}

Objectives: Hypertension is well established as a major risk factor for cardiovascular disease. Although there is undeniable evidence to support the beneficial effects of antihypertensive therapy on morbidity and mortality, adequate blood pressure management still remains suboptimal. Research into the treatment of hypertension has produced a multitude of drug classes with different efficacy profiles. These agents include $\beta$-blockers, diuretics, ACE inhibitors, angiotensin receptor blockers and calcium channel blockers. One of the oldest groups of antihypertensives, the calcium channel blockers are a heterogeneous group of medications.

Methods: This review paper will focus on amlodipine, a dihydropyridine calcium channel blockers, which has been widely used for 2 decades.

Results: Amlodipine has good efficacy and safety, in addition to strong evidence from large randomised controlled trials for cardiovascular event reduction. Conclusions: Amlodipine should be considered a first-line antihypertensive agent.

\section{INTRODUCTION}

Calcium channel blockers (CCBs) were first introduced over 35 years ago initially for coronary heart disease (CHD), but they soon gained wide recognition for their efficacy in hypertension (HTN). The initial indication, besides HTN, also included angina, peripheral vascular disease and some arrhythmic conditions. ${ }^{1}$ Amlodipine has many unique qualities that set it apart from other agents in this class. The aim of this review is to compare amlodipine with other antihypertensive agents with particular focus on the ability to improve cardiovascular (CV) health and reduce adverse $\mathrm{CV}$ outcomes.

\section{CLINICAL INDICATIONS,} PHARMACODYNAMICS AND PHARIMACOKINETICS

Amlodipine is a long-acting, lipophilic, third generation dihydropyridine (DHP) CCBs that exerts its action through inhibition of

\section{KEY QUESTIONS}

What is already known about this subject?

- It is well known that many antihypertensive medications reduce the risk of stroke and heart attack. This is generally believed to be a class effect however.

What does this study add?

- This review paper focusing on amlodipine showing how its unique properties may provide enhanced cardiovascular protections compared with other antihypertensive agents.

How might this impact on clinical practice?

- This paper sheds light on the evidence in support of amlodipine as a first-line antihypertensive agent for the prevention of strokes in particular.

calcium influx into vascular smooth muscle cells and myocardial cells, which results in decreased peripheral vascular resistance (PVR). Amlodipine is indicated for the treatment of high blood pressure (BP)/HTN and angina. In addition, a number of randomised trials have ascertained its utility in angina pectoris. $^{2}$ Amlodipine is usually dosed on a once daily basis because of its long half-life, which is favourable for patient compliance. A starting dose of $5 \mathrm{mg}$ is usually recommended with a maximum daily dose of $10 \mathrm{mg}$. In the elderly population and those with hepatic failure, a starting dose of $2.5 \mathrm{mg}$ is recommended. Amlodipine has a gradual onset of action and hence no significant reflex neuroendocrine activation. Activating reflex mechanisms, such as increased PVR and elevated heart rate, can cause negative effects on lipid and carbohydrate metabolism. These notable adverse effects are commonly seen with other agents including the first generation $\beta$-blockers (BBs; such as atenolol and metoprolol) and earlier generation of DHPs. Amlodipine has a high bioavailability, ranging from $60 \%$ to $80 \%$; it undergoes hepatic metabolism and shows some 
impaired elimination in the setting of liver cirrhosis but no accumulation with renal failure. Amlodipine also has a slow rate of elimination over 40-60 hours. If amlodipine is discontinued, BP generally returns to baseline over 1 week without any dangerous rebound elevations in BP (unlike clonidine). ${ }^{3}$

\section{SIDE EFFECT PROFILE}

The most commonly reported adverse effect hindering compliance with amlodipine is peripheral oedema. However, this adverse effect can be minimised if the agent is given at bedtime, and lower doses (2.5 or $5 \mathrm{mg} /$ day) are used. Indeed, the bedtime administration of nifedipine gastrointestinal therapeutic system was associated with a $93 \%$ reduction in oedema compared with morning dosing ( $1 \%$ vs $13 \%, \mathrm{p}<0.001$, respectively). ${ }^{4}$ Other reported side effects include dizziness, fatigue, headache, palpitations and nausea, although these are generally not bothersome enough to cause discontinuation of the drug. Amlodipine is contraindicated in breastfeeding women, cardiogenic shock and unstable angina. Also, its vasodilatory effect can lead to decreased cardiac output in the setting of aortic stenosis.

\section{ROLE AS MONOTHERAPY IN HTN}

Several HTN trials have investigated the efficacy of amlodipine monotherapy versus other agents, including diuretics, ACE inhibitors (ACEIs) and angiotensin receptor blockers (ARBs). The outcomes of these trials suggest that amlodipine has a neutral effect on several preexisting comorbid states, which will be discussed below.

The Comparison of Amlodipine versus Enalapril to Limit Occurrence of Thrombosis (CAMELOT) trial, enrolled 1991 patients with angiographically documented coronary artery disease (CAD) and randomised them to amlodipine $10 \mathrm{mg}$, enalapril $20 \mathrm{mg}$, or placebo and followed them over 24 months. Atherosclerotic progression was also assessed in a substudy of 274 patients as well using intravascular ultrasound. Although the baseline BP was low to begin with, 129/78, both groups showed similar BP lowering, 4.8/2.5 and 4.9/2.4 for amlodipine and enalapril, respectively. Amlodipine significantly reduced non-fatal myocardial infarction (MI) by $26 \%$ and stroke or transient ischaemic attack by $50 \%$ (number needed to treat $=16$ ), whereas enalapril had no significant benefit compared with placebo. ${ }^{5}$ Moreover, there was a statistically significant reduction in hospitalisation rate for angina $(\mathrm{p}=0.003)$ with amlodipine versus enalapril. This study suggests that normotensive patients treated with amlodipine show reduced rates of CV events and hospitalisations compared with enalapril and evidence of slowing of atherosclerotic progression. ${ }^{5}$

A Japanese study, although small, investigated the effect of losartan and amlodipine on left ventricular (LV) diastolic function in patients with mild-to-moderate HTN. LV diastolic dysfunction has a direct correlation with LV hypertrophy (LVH) and myocardial fibrosis.
Previous trials have established the beneficial role of ACEIs on cardiac remodelling but other studies with ARBs versus amlodipine showed a greater benefit in the CCB class. Thus, the effect of losartan and amlodipine on LV diastolic function and atherosclerosis in Japanese patients with mild-to-moderate HTN (J-ELAN) trial was undertaken to compare the effects of an ARB (losartan) versus a CCB (amlodipine) on LV diastolic dysfunction. J-ELAN enrolled 57 patients with LVH and mild-to-moderate HTN and randomised them to either losartan or amlodipine. ${ }^{6}$ The doses of these drugs were uptitrated over an 18-month period and then other anti-HTN agents were added, to achieve goal BP control, except those agents that affect LVH such as ACEIs, ARBs, other CCBs or BBs. Both groups showed similar reduction in BP but the losartan group had a greater effect on carotid intimal-medial thickness. ${ }^{7}$ In addition, there was no significant difference in terms of LV mass in the two groups. These findings suggest that amlodipine is non-inferior to ARBs with respect to its effects on LV remodelling. ${ }^{6} 7$

The ALLHAT (Antihypertensive Lipid-Lowering Treatment to Prevent Heart Attack Trial) was one of the largest $\mathrm{BP}$ trials performed to date, which enrolled over 33000 patients with HTN and one CHD risk factor. The objective of this landmark trial was to determine whether the incidence of CHD or other CV diseases would be lower in those patients treated with a CCB, an ACEI, or a diuretic. Patients were randomised to chlorthalidone, amlodipine, or lisinopril with a mean follow-up of about 4.9 years. ${ }^{8}$ Primary outcomes included fatal CHD or non-fatal MI and secondary outcomes included all-cause mortality. There was no significant difference among the groups for the reduction in the primary or secondary outcome of all-cause mortality. It was determined that thiazide diuretics are superior in preventing one or more forms of CV disease and also since they are less expensive should be a first-line agent in the treatment of HTN. However, other commentaries suggested that this finding may be attributed to the fact that thiazides are better at treating volume-dependent HTN, such as the elderly and African-Americans who comprised a large portion of the patient demographics for this study. ${ }^{8-10}$ Although this may be the case, this very large study showed that amlodipine was neither superior nor inferior to thiazide diuretics or ACEIs in managing HTN in patients with other comorbid conditions. ${ }^{10}$

Studies have shown that nitric oxide (NO) production is diminished in patients with HTN. ${ }^{11}{ }^{12}$ A small study carried out by Masayoshi and colleagues measured exhaled NO in seven previously untreated participants to assess whether amlodipine has an effect on NO. ${ }^{13}$ Their study found that NO production in the pulmonary circulation was increased as evidenced by increased NO measurements in exhaled air after 2 months of amlodipine therapy. Another small study by Zhang and colleagues evaluated NO production in explanted hearts, 
which were harvested during transplant. Previous studies suggest that NO release from endothelial cells is a kininmediated mechanism. Kinins are usually degraded by ACE. ACEIs facilitate the accumulation of these compounds, which was the rational for enlisting ramiprilat for comparison. ${ }^{14}$ While amlodipine was found to increase NO production in these failing hearts, it was similar to the NO production noted with ramiprilat. The authors postulated that this may be one of the mechanisms of amlodipine's beneficial effects in heart failure (HF), which is not a feature shared by other members of the CCB class. Thus, the enhancement of NO production may account for the beneficial effects of this drug on the CV system. ${ }^{14}$ Additionally, amlodipine has anti-inflammatory and antioxidative effects giving it vasoprotective effects beyond its BP-lowering benefits. ${ }^{15}$ Interestingly, these benefits were noted by the authors to be caused by an increase in endothelial NO synthase expression and inhibition of ACE. Thus, amlodipine may even be beneficial for patients with high renin HTN. $^{15}$

The VALUE (Valsartan Antihypertensive Long-term Use Evaluation) trial was another large trial enrolling over 15000 patients with HTN who were previously treated with anti-HTN therapy. ${ }^{16}$ This study looked at the efficacy of amlodipine versus valsartan in attaining a BP goal of $<140 / 90$. The doses were uptitrated to $10 \mathrm{mg}$ of amlodipine or $160 \mathrm{mg}$ of valsartan. Two additional steps were done that included adding hydrochlorothiazide (HCTZ) then another agent (excluding ACEIs, ARBs or other CCBs). The outcomes showed that both monotherapy groups had similar improvements in BP with average BPs in the $130 \mathrm{~s} / 80 \mathrm{~s}$ for both groups. However, valsartan caused a significant increase (19\%, $\mathrm{p}=0.02$ ) in total MI (fatal and nonfatal) in comparison with amlodipine. Although some have criticised these results due to the faster BP lowering of amlodipine early on, the Kaplan-Meier MI curves indicated that as BP became similar between the two groups (as the trial progressed), the curves continued to diverge; suggesting a BP-independent beneficial effect on MI with amlodipine versus valsartan. New-onset diabetes was reported in 580 $(11.5 \%)$ patients on valsartan and in 718 (14.5\%) patients on amlodipine. ${ }^{1617}$

The Fosinopril versus Amlodipine Cardiovascular Events Randomized Trial (FACET) showed different results on fasting glucose levels. The goal of the FACET was to compare the effects of these two agents on lipid and diabetes control in patients with HTN. ${ }^{18}$ Three hundred and eighty patients were randomised to $20 \mathrm{mg}$ of fosinopril or $10 \mathrm{mg}$ of amlodipine daily with a follow-up period of 3.5 years. Results showed no difference on the lipid profiles or fasting glucose levels. However, fosinopril was associated with a $38.5 \%$ relative risk reduction (RRR) in the combined end point (fatal or non-fatal stroke and fatal or non-fatal MI; $R R=0.6151$, 95\% CI 0.3266 to 1.1587 ). These findings suggest that while amlodipine may have similar effects on BP control, fosinopril may have a greater benefit for the reduction of stroke/MI. However, this trial enrolled a small number of patients and the thus the CIs were wide and the $\mathrm{CV}$ event reduction with fosinopril was not significant compared with amlodipine. A larger trial is needed to clarify these results. ${ }^{18}$

Another study by Mugellini and colleagues looked at 391 patients with metabolic syndrome who had at least two episodes of atrial fibrillation (AF) in 6 months. ${ }^{19}$ These patients were randomised to telmisartan, ramipril or amlodipine for 12 months and results showed that all three groups similarly reduced both systolic and diastolic BP. In terms of the AF however, telmisartan showed the greatest effect on reducing recurrence. ${ }^{19}$

\section{ATHEROSCLEROSIS AND CAD}

Calcium regulation has been implicated in the pathogenesis of atherosclerotic plaque formation, which has generated interest in the potential role for this CCB in the prevention of atherosclerosis. ${ }^{20}$ Several mechanisms have been proposed to account for amlodipine's potential benefit in atherosclerosis. In vivo and in vitro studies suggest that amlodipine inhibits oxidative damage to the lipid bilayer of the cell membrane and this has been attributed to its lipophilicity and chemical structure, which prevents the formation of free radicals. This process is prevented by the donation of protons by this drug to lipid peroxide molecules. In atherogenesis, the packing of phospholipid molecules becomes disarrayed and this causes swelling of the lipid bilayer, which is an impetus for smooth muscle proliferation and atheroma development. Enhancement of NO production has been proposed as a potential reason for amlodipine's antiatherosclerotic effect. Zhang and Hintze ${ }^{21}$ noted increased NO production in canine coronary microvasculature, which was an unexpected feature of this CCB and is not a quality that is shared by other members of its class. Additionally, amlodipine has been shown to upregulate the expression of interleukins, which may also have antiproliferative effects, and to have favourable effects on extracellular matrix remodelling. ${ }^{22} 23$

One of the pivotal trials assessing amlodipine's role in atherosclerosis was the Prospective Randomized Evaluation of the Vascular Effects of Norvasc (PREVENT) trial. PREVENT assessed the development and progression of atherosclerosis in patients with known CAD. This was a multicentre, randomised, placebo-controlled, double-blinded study, which looked at 825 patients with angiographically documented CAD. ${ }^{20}$ Patients were followed over a 3 -year period and the primary end point was change in mean diameter over 36 months in segments with a baseline of $30 \%$ stenosis. It has been suggested that acute coronary syndrome does not usually result from more stable plaques but rather rupture of minimal lesions. PREVENT also assessed the rate of progression of carotid atherosclerosis which was assessed by CIMT. The data showed that there 
was no effect on the risk of all-cause mortality or major CV events, and amlodipine did not alter the development of or slow the progression of CAD lesions. However, there was a statistically significant effect on the progression of carotid artery atherosclerosis $(p=0.007){ }^{24}$ Yet, the study had low statistical power for the detection of a treatment difference in mortality and major morbidity rates because of the relatively low event rates (ie, $<2 \% /$ year for MI). When major and other documented vascular events/procedures were combined (increasing the power to determine an effect), there were fewer events in the amlodipine group. Additionally, amlodipine did show reduced rates of unstable angina and coronary revascularisation, which is comparable to data seen with treatment with BB, nitrates and lipid-lowering agents. Angiographic trials with nifedipine or nicardipine did not show these effects. The authors of PREVENT did report that when the event rates for unstable angina and coronary revascularisation were assessed closely, these curves diverged very early (in the first year). Thus, while amlodipine may not have shown a significant role in prevention of progression of early coronary atherosclerotic lesions, it does have a beneficial effect in reducing hospitalisation rates for angina and coronary revascularisation. ${ }^{24}$ In the randomised trial Coronary Angioplasty Amlodipine Restenosis Study (CAPARES), patients had a reduced incidence of repeat percutaneous transluminal coronary angioplasty when treated with amlodipine. ${ }^{25}$

The beneficial effects of amlodipine go beyond calcium channel blockade for HTN management. Although many of these trials show that amlodipine is not superior to other agents in preventing $\mathrm{CAD}$, it can be safely used in patients with CAD for the management of HTN.

\section{ROLE IN RENOPROTECTION}

In the ACCOMPLISH trial, HCTZ was inferior to amlodipine for the prevention of end-stage renal disease. Furthermore, in patients who were $>65$ years old at baseline, there was a $70 \%$ RRR in patients progressing to dialysis in the amlodipine group versus the HCTZ group $\left(\mathrm{p}=0.053\right.$, for the difference) ${ }^{26}$ In the intention-to-treat population, the amlodipine group had a $48 \%$ RRR for chronic kidney disease (CKD) progression, defined as doubling of serum creatinine, estimated glomerular filtration rate (eGFR) $<15 \mathrm{~mL} / \mathrm{min}$, or dialysis compared with the HCTZ group. Furthermore, patients with CKD, defined as an eGFR of $45.1 \mathrm{~mL} / \mathrm{min}$ at baseline, showed a significantly greater decline in renal function with HCTZ versus amlodipine $(-2.3$ vs $-1.6 \mathrm{~mL} / \mathrm{min}$; $\mathrm{p}=0.001){ }^{26} 27$

\section{ROLE IN HF}

Traditionally CCBs were discontinued for the treatment of angina or HTN in patients with HF. Previous studies with CCBs such as nifedipine, have shown unfavourable effects due to reflex stimulation of the sympathetic nervous system and negative inotropic effects, features not seen with amlodipine. ${ }^{28-30}$ Thus, amlodipine may be safe in patients with HF. ${ }^{31}$ The Prospective Randomized Amlodipine Survival Evaluation (PRAISE) trial established the safety of amlodipine for angina and HTN in patients with advanced LV dysfunction (amlodipine). ${ }^{32}$ The PRAISE trial studied over 1100 patients with severe ischaemic and non-ischaemic New York Heart Association class III and IV HF with an LV ejection fraction (LVEF) of $<30 \%$ despite being on digoxin, diuretics or an ACEI. They were also allowed to be on a nitrate but no other vasodilating therapy. They were randomised to either amlodipine or placebo and followed for 33 months. There was no significant difference in the primary end point (combined risk of all-cause mortality and CV disease morbidity) with amlodipine treatment; although amlodipine did show a $9 \%$ risk reduction of a primary fatal or non-fatal event. There was a difference however, in the non-ischaemic HF group, where amlodipine showed a $46 \%$ reduction in mortality. ${ }^{32}$

The PRAISE trial showed that amlodipine did not affect the natural history of HF or increase the risk of death as other trials with CCBs have suggested. ${ }^{31}$ The investigators pointed out that it was interesting that amlodipine seemed to greatly benefit the non-ischaemic group. The PRAISE II trial was then carried out to further investigate the potential for amlodipine to reduce mortality in the non-ischaemic group. This trial looked at 1652 patients with normal arteriograms but with the systolic $\mathrm{HF}$ and average LVEF was $21 \%$. There was no significant difference found between the amlodipine versus placebo group in terms of all-cause or CV disease mortality or CV disease events. Taken together, PRAISE I and II suggest that amlodipine can be safely used to treat angina or HTN in patients with coexisting HF. ${ }^{33} 34$

\section{ROLE IN COMBINATION THERAPY FOR HTN}

While thus far amlodipine has shown to be non-inferior to many other anti-HTN therapies, the focus on HTN treatment seems to be shifting more towards combination therapy. Less than $50 \%$ of patients with stage I or II HTN are adequately controlled with monotherapy, and thus initial treatment for HTN in the majority of patients will require two agents. The classical approach to treating HTN where one first-line agent is maximised before another is added may be flawed since this is a multifactorial disease often occurring with a number of comorbidities. Many studies show that individuals with more CV risk factors generally need more anti-HTN agents to successfully manage their BP. Combination therapy using different mechanisms can lead to more effective BP lowering. In addition, combination therapies block counter-regulatory mechanisms that often limit the efficacy of monotherapy. Furthermore, using multiple agents to lower doses may reduce side effects, and thus improve patient compliance. 
The issue arises as to which fixed combinations provide the most benefits. Thiazides in combination with ACEIs or ARBs have synergistic BP-lowering activity, whereas thiazides and BBs have deleterious effects on the metabolic profile. CCBs are powerful vasodilators but can result in renin-angiotensin-aldosterone system (RAAS) activation, thus an ACEI or ARB counteracts this mechanism and leads to enhanced antihypertensive effect. Growing evidence from trial data also shows that CCBs with ACEIs or ARBs may provide the best longterm outcomes.

The ACCOMPLISH trial was a randomised, controlled trial in which 11506 patients with HTN with high risk for adverse CV events were assigned amlodipine+benazepril versus HCTZ+benazepril. ${ }^{35}$ The primary end point was the composite of $\mathrm{CV}$ death or major adverse $\mathrm{CV}$ events. The amlodipine+benazepril combination was superior to the HCTZ+benazepril for lowering CV death and adverse events (figure 1). ${ }^{35}$

The Anglo-Scandinavian Cardiac Outcomes Trial (ASCOT) included a total of 19257 patients with HTN (baseline BP, 164/95 mm Hg) and at least three other cardiac risk factors. ${ }^{36}$ This trial, which was stopped for benefit after 5.5 years, showed that the amlodipine +perindopril versus atenolol+thiazide diuretic significantly reduced all-cause mortality (RRR, $11 \%$; $\mathrm{p}=0.0247$; figure 2). Additionally, amlodipine+perindopril reduced CV mortality by $24 \%(\mathrm{p}=0.001)$, coronary events by $13 \%$ $(\mathrm{p}=0.007)$ and strokes by $23 \% \quad(\mathrm{p}=0.0003) .{ }^{37}$ At the point where rates of $\mathrm{CV}$ death diverged (red arrow) most of the patients $(78 \%)$ were treated with the combination of perindopril+amlodipine rather than amlodipine monotherapy. ${ }^{36}$

The Assessment of combination Therapy of Amlodipine/Ramipril (ATAR) study was an 18-week randomised prospective double-blinded Brazilian study which compared the combination of amlodipine and ramipril versus amlodipine monotherapy. The mean changes in ambulatory BP measurements were statistically significant between the combination versus the amlodipine monotherapy group, $18.7 \%$ vs $7.6 \%$, $\mathrm{p}=0.011$, respectively. In addition, the reported incidence of peripheral oedema was lower in the combination group. ${ }^{38}$

The Candesartan and Diuretic versus Amlodipine in hypertensive patients (CANDIA) trial evaluated candesartan+HCTZ combination versus amlodipine monotherapy. ${ }^{39}$ This multicentre, double-blinded, randomised trial assessed patients with mild-to-moderate HTN not adequately controlled with monotherapy. After 8 weeks of therapy, there was no significant difference between the two groups. Systolic BP decreased by about $15 \mathrm{~mm} \mathrm{Hg}$; however, there was a higher discontinuation rate with amlodipine versus the combination drug due to peripheral oedema, $18 \%$ vs $6 \%$, respectively. These findings suggest that while both agents are effective in lowering BP, the candesartan+HCTZ combination was better tolerated and hence may lead to better patient compliance. $^{39}$

The Combination of Olmesartan Medoxomil and Amlodipine Besylate in Controlling High blood Pressure (COACH) study was a study evaluating an ARB+amlodipine combination versus placebo. ${ }^{40}$ The 1940 patients in this 8-week treatment study showed achievement of BP goals $(<140 / 90)$ with the combination therapy and again, a lower incidence of peripheral oedema. ${ }^{40}$ These findings were echoed in the Telmisartan plus Amlodipine Study in amlodipine $5 \mathrm{mg}$ (TEAMSTA-5), which tested another ARB+amlodipine combination. The study showed that the combination of telmisartan $40 / 80 \mathrm{mg}$ plus amlodipine $5 \mathrm{mg}$ was superior to amlodipine $10 \mathrm{mg}$ monotherapy. ${ }^{41}$

CCBs are potent vasodilators and hence reduce $\mathrm{BP}$ effectively but can result in RAAS activation. Therefore, the rational for a CCB/RAAS inhibitor combination drug would theoretically provide vasodilation while buffering RAAS activation. Another combination with RAAS blocker and HCTZ has been shown to be effective. Triple therapy with valsartan+amlodipine+HCTZ was compared with dual combination with valsartan+HCTZ,
Figure 1 ACCOMPLISH: the amlodipine+benazepril combination lowered the primary end point by a relative risk reduction was $20 \%$ (HR 0.80 ; $95 \% \mathrm{Cl} 0.72$ to $0.90 ; p<0.001){ }^{35}$

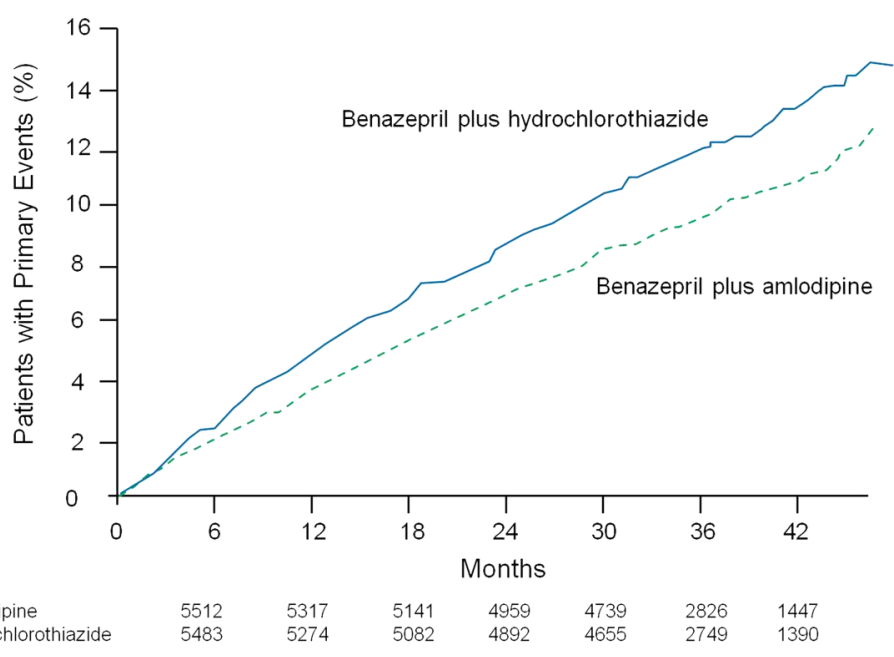




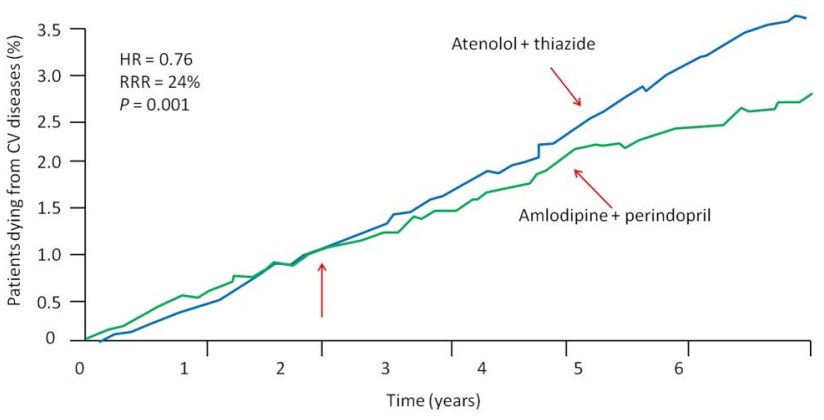

Figure 2 ASCOT: randomised trial showing a decrease in cardiovascular mortality in patients treated with amlodipine/ perindopril compared with atenolol/thiazide therapy. ${ }^{37}$ ASCOT, Anglo-Scandinavian Cardiac Outcomes Trial; CV, cardiovascular; RRR, relative risk reduction.

amlodipine+valsartan or amlodipine+HCTZ. This was a randomised, double-blinded study in 2271 patients with moderate-to-severe HTN. The goal was to attain a BP goal of $<140 / 90$. The outcomes showed that this triple therapy combination was superior to any of the dual combination drugs at reducing sitting systolic and diastolic BP $(p<0.0001)$. The more pronounced findings were in those with higher baseline BP and the results were consistent over all ages, genders and ethnicities. The adverse events reported were similar across all treatment groups and included peripheral oedema as the most common, along with headache and dizziness. ${ }^{42}$

\section{CONCLUSION}

Amlodipine is an excellent first-line choice among the myriad options of antihypertensive agents. In the studies discussed in this manuscript, amlodipine was highly effective for the treatment of HTN and stable angina as evidenced by the fewer hospitalisations for unstable angina and revascularisation in randomised controlled trials. Amlodipine has also shown robust reductions on CV end points (especially stroke) but has not altered the prognosis in HF. Its abilities to prevent activation of counter-regulatory mechanisms, to slow the progression of atherosclerosis, to confer antioxidant properties and to enhance NO production are all unique actions. The management of HTN is shifting more towards dual or even triple combination therapy and requires a patient profiling approach as the number of comorbid states increases. Amlodipine is a superior option in the HTN armamentarium, not only for controlling BP but also for safely improving patient outcomes.

Contributors HF assisted with the gathering and review of the data; JJD, JHO and CJL reviewed the data; HF, JJD, JHO and CJL assisted in the concept and design of the manuscript. HF, JJD, JHO and CJL wrote, rewrote and finalised the manuscript.

Funding This manuscript received no specific grant from any funding agency in the public, commercial, or not-for-profit sectors.

Competing interests None declared.
Provenance and peer review Not commissioned; externally peer reviewed.

Data sharing statement No additional data are available.

Open Access This is an Open Access article distributed in accordance with the Creative Commons Attribution Non Commercial (CC BY-NC 4.0) license, which permits others to distribute, remix, adapt, build upon this work noncommercially, and license their derivative works on different terms, provided the original work is properly cited and the use is non-commercial. See: http:// creativecommons.org/licenses/by-nc/4.0/

\section{REFERENCES}

1. Triggle DJ. Calcium channel antagonists: clinical uses-past, present and future. Biochem Pharmacol 2007;74:1-9.

2. Taylor $\mathrm{SH}$. Usefulness of amlodipine for angina pectoris. Am J Cardiol 1994;73:28A-33A.

3. Abernethy DR. Pharmacokinetics and pharmacodynamics of amlodipine. Cardiology 1992;80(Suppl 1):31-6.

4. Hermida RC, Ayala DE, Mojón A, et al. Chronotherapy with nifedipine GITS in hypertensive patients: improved efficacy and safety with bedtime dosing. Am J Hypertens 2008;21:948-54.

5. Nissen SE, Tuzcu EM, Libby $P$, et al. Effect of antihypertensive agents on cardiovascular events in patients with coronary disease and normal blood pressure: the CAMELOT study: a randomized controlled trial. JAMA 2004;292:2217-25.

6. Yamamoto K, Ozaki H, Takayasu K, et al. The effect of losartan and amlodipine on left ventricular diastolic function and atherosclerosis in Japanese patients with mild-to-moderate hypertension (J-ELAN) study. Hypertens Res 2011;34:325-30.

7. J-ELAN Investigators. Effect of losartan and amlodipine on left ventricular diastolic function in patients with mild-to-moderate hypertension (J-ELAN): rationale and design. Circ J 2006;70:124-8.

8. Chrysant SG. The ALLHAT study: results and clinical implications. QJM 2003;96:771-3.

9. Salvetti A, Ghiadoni L. Guidelines for antihypertensive treatment: an update after the ALLHAT study. J Am Soc Nephrol 2004;15(Suppl 1):S51-4.

10. The ALLHAT Officers and Coordinators for the ALLHAT Collaborative Research Group. Major outcomes in high-risk hypertensive patients randomized to angiotensin-converting enzyme inhibitor or calcium channel blocker vs diuretic: the Antihypertensive and Lipid-Lowering Treatment to Prevent Heart Attack Trial (ALLHAT). JAMA 2002;288:2981-97.

11. Calver A, Collier J, Vallance P. Forearm blood flow responses to a nitric oxide synthase inhibitor in patients with treated essential hypertension. Cardiovasc Res 1994;28:1720-5.

12. Camilletti A, Moretti N, Giacchetti G, et al. Decreased nitric oxide levels and increased calcium content in platelets of hypertensive patients. Am J Hypertens 2001;14(Pt 1):382-6.

13. Kato M, Matsumoto A, Nakajima $T$, et al. Amlodipine increases nitric oxide production in exhaled air during exercise in patients with essential hypertension. Am J Hypertens 2004;17:729-33.

14. Linz W, Wohlfart $P$, Schölkens BA, et al. Interactions among ACE, kinins and NO. Cardiovasc Res 1999;43:549-61.

15. Toba H, Nakagawa $\mathrm{Y}$, Miki S, et al. Calcium channel blockades exhibit anti-inflammatory and antioxidative effects by augmentation of endothelial nitric oxide synthase and the inhibition of angiotensin converting enzyme in the $\mathrm{N}(\mathrm{G})$-nitro-L-arginine methyl ester-induced hypertensive rat aorta: vasoprotective effects beyond the blood pressure-lowering effects of amlodipine and manidipine. Hypertens Res 2005;28:689-700.

16. Julius S, Kjeldsen SE, Weber M, et al. Outcomes in hypertensive patients at high cardiovascular risk treated with regimens based on valsartan or amlodipine: the VALUE randomised trial. Lancet 2004;363:2022-31.

17. Kjeldsen SE, Julius S, Mancia G, et al. Effects of valsartan compared to amlodipine on preventing type 2 diabetes in high-risk hypertensive patients: the VALUE trial. J Hypertens 2006;24:1405-12.

18. Tatti $P$, Pahor $M$, Byington RP, et al. Outcome results of the Fosinopril Versus Amlodipine Cardiovascular Events Randomized Trial (FACET) in patients with hypertension and NIDDM. Diabetes Care 1998;21:597-603.

19. Fogari R, Mugellini A, Zoppi A, et al. Effect of telmisartan and ramipril on atrial fibrillation recurrence and severity in hypertensive patients with metabolic syndrome and recurrent symptomatic paroxysmal and persistent atrial fibrillation. J Cardiovasc Pharmacol Ther 2012;17:34-43.

20. Mason RP. Mechanisms of atherosclerotic plaque stabilization for a lipophilic calcium antagonist amlodipine. Am J Cardiol $2001 ; 88: 2 M-6 M$. 
21. Zhang $X$, Hintze TH. Amlodipine releases nitric oxide from canine coronary microvessels: an unexpected mechanism of action of a calcium channel-blocking agent. Circulation 1998:97:576-80.

22. Eickelberg O, Roth M, Block LH. Effects of amlodipine on gene expression and extracellular matrix formation in human vascular smooth muscle cells and fibroblasts: implications for vascular protection. Int J Cardiol 1997;62(Suppl 2):S31-7.

23. Roth M, Eickelberg O, Kohler E, et al. Ca2+ channel blockers modulate metabolism of collagens within the extracellular matrix. Proc Natl Acad Sci USA 1996;93:5478-82.

24. Pitt $B$, Byington RP, Furberg CD, et al. Effect of amlodipine on the progression of atherosclerosis and the occurrence of clinical events. PREVENT Investigators. Circulation 2000;102:1503-10.

25. Mason RP. Mechanisms of plaque stabilization for the dihydropyridine calcium channel blocker amlodipine: review of the evidence. Atherosclerosis 2002;165:191-9.

26. DiNicolantonio JJ. Hydrochlorothiazide: is it a wise choice? Expert Opin Pharmacother 2012;13:807-14.

27. Bakris GL, Sarafidis PA, Weir MR, et al. Renal outcomes with different fixed-dose combination therapies in patients with hypertension at high risk for cardiovascular events (ACCOMPLISH) a prespecified secondary analysis of a randomised controlled trial. Lancet 2010;375:1173-81.

28. Goldstein RE, Boccuzzi SJ, Cruess D, et al. Diltiazem increases late-onset congestive heart failure in postinfarction patients with early reduction in ejection fraction. The Adverse Experience Committee; and the Multicenter Diltiazem Postinfarction Research Group. Circulation 1991;83:52-60.

29. Elkayam U, Amin J, Mehra A, et al. A prospective, randomized, double-blind, crossover study to compare the efficacy and safety of chronic nifedipine therapy with that of isosorbide dinitrate and their combination in the treatment of chronic congestive heart failure. Circulation 1990;82:1954-61.

30. Packer M, Lee WH, Medina N, et al. Prognostic importance of the immediate hemodynamic response to nifedipine in patients with severe left ventricular dysfunction. J Am Coll Cardiol 1987;10:1303-11.

31. Hoyt RE, Bowling LS. Reducing readmissions for congestive heart failure. Am Fam Physician 2001;63:1593-8.

32. O'Connor CM, Carson PE, Miller AB, et al. Effect of amlodipine on mode of death among patients with advanced heart failure in the
PRAISE trial. Prospective Randomized Amlodipine Survival Evaluation. Am J Cardiol 1998:82:881-7.

33. Thackray $\mathrm{S}$, Witte $\mathrm{K}$, Clark $\mathrm{AL}$, et al. Clinical trials update: OPTIME-CHF, PRAISE-2, ALL-HAT. Eur J Heart Fail 2000;2:209-12.

34. Packer M, Carson P, Elkayam U, et al. Effect of amlodipine on the survival of patients with severe chronic heart failure due to a nonischemic cardiomyopathy: results of the PRAISE-2 study (prospective randomized amlodipine survival evaluation 2). JACC Heart Fail 2013;1:308-14.

35. Jamerson K, Weber MA, Bakris GL, et al. Benazepril plus amlodipine or hydrochlorothiazide for hypertension in high-risk patients. N Engl J Med 2008;359:2417-28.

36. Dahlof B, Sever PS, Poulter NR, et al. Prevention of cardiovascular events with an antihypertensive regimen of amlodipine adding perindopril as required versus atenolol adding bendroflumethiazide as required, in the Anglo-Scandinavian Cardiac Outcomes Trial-Blood Pressure Lowering Arm (ASCOT-BPLA): a multicentre randomised controlled trial. Lancet 2005;366:895-906.

37. Clavreul N. Optimizing combination therapy for cardiovascular protection: evidence from landmark trials. Medicographia 2013;35:455-63.

38. Miranda RD, Mion D Jr, Rocha JC, et al. An 18-week, prospective, randomized, double-blind, multicenter study of amlodipine/ramipril combination versus amlodipine monotherapy in the treatment of hypertension: the assessment of combination therapy of amlodipine/ ramipril (ATAR) study. Clin Ther 2008;30:1618-28.

39. Fogari R, Mugellini A, Derosa G. Efficacy and tolerability of candesartan cilexetil/hydrochlorothiazide and amlodipine in patients with poorly controlled mild-to-moderate essential hypertension. $J$ Renin Angiotensin Aldosterone Syst 2007;8:139-44

40. Chrysant SG, Melino M, Karki S, et al. The combination of olmesartan medoxomil and amlodipine besylate in controlling high blood pressure: $\mathrm{COACH}$, a randomized, double-blind,

placebo-controlled, 8-week factorial efficacy and safety study. Clin Ther 2008;30:587-604

41. Neldam S, Lang M, Jones R. Telmisartan and amlodipine single-pill combinations vs amlodipine monotherapy for superior blood pressure lowering and improved tolerability in patients with uncontrolled hypertension: results of the TEAMSTA-5 study. J Clin Hypertens (Greenwich) 2011;13:459-66.

42. Calhoun DA, Lacourcière $\mathrm{Y}$, Chiang YT, et al. Triple antihypertensive therapy with amlodipine, valsartan, and hydrochlorothiazide: a randomized clinical trial. Hypertension 2009;54:32-9. 